\title{
Y avait-il antéposition stylistique en ancien français ?
}

\author{
Labelle, Marie \\ Université du Québec à Montréal \\ Hirschbühler, Paul \\ Professeur retraité de l'université d'Ottawa
}

Un coup de dés jamais n'abolira le hasard (Mallarmé, 1897)

\section{Antéposition stylistique}

L'ancien français est généralement considéré comme une langue à verbe second (V2) non strict dans les propositions principales. Dans les principales déclaratives, le verbe tensé occupe généralement la seconde position, précédé d'un expression simple ou complexe, mais il peut également occuper une position plus en retrait, ou encore la position initiale. ${ }^{1}$ Pour leur part, les subordonnées tendent à être de type SVO, mais, à nouveau, d'autres ordres sont également attestés, notamment l'ordre XV(S) typique des principales est observé dans les subordonnées complétives introduites par certaines classes de verbes, comme les verbes déclaratifs (cf. Vance 1997, chap. 4). Ce type de V2 en subordonnée est bien attesté, sous une variété de conditions, dans les langues germaniques et scandinaves (Heycock 2006), et nous n'en parlerons pas ici. Notre objectif dans ce travail est d'étudier un autre type d'écart par rapport à l'ordre SVO en subordonnée, celui où certaines catégories lexicales (verbe non tensé, adverbe, adjectif) ou syntagmes précèdent le verbe, y compris dans des types subordonnées qui ne permettent pas la construction V2, comme les propositions relatives, les interrogatives indirectes ou les subordonnées temporelles. $^{2}$

(1) Einsint vint à .I. parlement a Soissons qui nomez fu, ...

'Ainsi il vint à une assemblée à Soissons qui fut convoquée, ...' [CONSTANTINOPLE, p. 60]

(2) Si jurroient li compaignon tel serement come cil font qui en queste doivent entrer

'Les compagnons prêteraient le serment habituel de ceux qui doivent entrer dans une quête.'

[QUESTE §32, in Mathieu 2006a ex. (1)]

(3) Qant levé furent del mangier,...

'Quant ils eurent terminé le repas ...'

[CHARRETTE, 1043, in Mathieu 2006a ex. (7a)]

Sur la base de la ressemblance entre (1)-(3) et la construction d'antéposition stylistique (terme introduit par Mathieu pour traduire Stylistic Fronting, $S F$ ) de l'islandais illustrée en (4), plusieurs auteurs ont proposé que l'antéposition stylistique existait également en ancien français (Cardinaletti \& Roberts, 1991/2002 ; Dupuis, 1989 ; Mathieu 2006a, 2006b, 2009, 2013 ; Molnár, 2010 ; Roberts 1993).

(4) Hver heldur pú að stolið hafi hjólinu? (Holmberg, 2006 : ex. 10b)

qui pensez vous que volé a bicyclette-DEF

'Qui pensez-vous qui a volé la bicyclette?' 
L'antéposition stylistique est une construction de l'islandais décrite par Maling (1980/1990, ci-après Maling 1990), distincte de l'antéposition syntaxique de l'élément initial d'une construction V2, que nous appellerons, dans le présent travail antéposition $V 2 .{ }^{3}$ Les principales différences entre les deux constructions seront mentionnées en 2.1. L'antéposition stylistique a suscité de multiples analyses qui constituent un sujet de controverses (cf., entre autres, Holmberg 2006, 2013 pour un état de la question et Hrafnbjargarson 2004 pour une vue divergente des analyses les plus communément admises pour l'islandais). Plusieurs auteurs ont proposé que cette construction se retrouve dans d'autres langues romanes médiévales ou modernes du fait de certaines similitudes avec la construction de l'islandais, malgré l'observation de différences (Benincà 2006 ; Cardinaletti 2003 ; Egerland 2011 ; Fischer 2010, 2014 ; Fischer et Alexiadou 2001 ; Fontana 1993 ; Franco 2009, 2012 ; Martins 2005, 2011). Salvesen (2011, 2013) recourt également à cette étiquette pour l'ancien français, mais distingue clairement l'antéposition de l'ancien français du processus typique de l'islandais et des langues scandinaves anciennes.

L'hypothèse que la construction en (1)-(3) est la même que celle illustrée en (4), mise en doute par Labelle $(2007,2013)$ et Zaring (2011a, 2011b) pour la période la plus ancienne de l'ancien français, a été réitérée récemment par Mathieu (2013).

Le but du présent travail est de reprendre la question et de montrer que, malgré un air de famille, la construction en (1)-(3) de l'ancien français a des propriétés suffisamment différentes de celles de l'islandais pour que l'on distingue les deux et que l'on ne parle pas, dans le cas de l'ancien et du moyen français, d'une construction d'antéposition stylistique. Pour l'ancien français, nous parlerons de déplacement stylistique à gauche (DSG), un terme purement descriptif, et nous montrerons que cette expression recouvre plusieurs constructions.

Pour des raisons d'espace, et parce que l'antéposition stylistique est mieux documentée pour les subordonnées et y est plus facile à distinguer de l'antéposition V2, nous nous limitons ici aux subordonnées, bien que Mathieu (2006a, 2013) étende l'analyse à certaines principales, notamment dans le cas de l'antéposition d'un participe.

\section{L'antéposition stylistique versus le déplacement stylistique à gauche}

\subsection{L'antéposition stylistique en islandais}

Les subordonnées de l'islandais sont normalement de type SVO, mais certains processus sont la source d'autres ordres. Ainsi, l'antéposition stylistique permet l'apparition d'une tête ou d'un constituant entre l'introducteur de subordonnée (complémenteur, relatif, expression interrogative) et le verbe tensé lorsque le sujet n'occupe pas cette position (Maling 1980/1990). L'ordre obtenu rappelle la construction V2. Mais, en plus de se produire dans des propositions où V2 en subordonnée n'est pas permis (p. ex. relatives, interrogatives indirectes), l'antéposition stylistique se distingue par diverses propriétés de l'antéposition V2 (cf. Bailyn 2004 ; Fisher \& Alexiadou 2001 ; Holmberg 2006, 2013 ; Maling 1990 ; Ott 2009) :

1) Selon Maling (1990), le constituant initial d'une phrase V2 est associé à un effet d'emphase ou de focalisation qu'on ne trouve pas dans l'antéposition stylistique.

2) Les éléments sujets à l'antéposition stylistique et à l'antéposition V2 sont partiellement distincts (Holmberg 2000). Les NP, PP, adjectifs prédicatifs et certains adverbes peuvent apparaitre en position initiale dans les deux cas. Mais l'antéposition stylistique permet également le déplacement de têtes (particules verbales, participes, négation), qui ne sont pas typiques de l'antéposition V2.

3) En général, l'antéposition stylistique favorise les constituants phonologiquement "légers", ce qui n'est pas le cas dans V2 (Wood 2011). C'est peut-être ce qui fait qu'un NP/DP complexe (5), une proposition ou un VP (6) ne peuvent pas être antéposés (Ott 2009: 149-150, ex. (21a), (22a)) : 
(5) *Allir sem [NP eyðileggingu borgarinnar Hirosima $]_{\mathrm{i}}$ fengu að fylgjast með $t_{\mathrm{i}}$ fylltust Tous qui la destruction.DAT la ville.GEN Hiroshima purent suivre avec remplis hryllingi terreur 'tous ceux qui ont pu suivre la destruction de la ville d'Hiroshima étaient remplis de terreur.'

(6) *peir sem [vp búið í Ósló $]_{i}$ hafa $t_{\mathrm{i}}$ segja að... ceux qui [vp vécu à Oslo $]_{\mathrm{i}}$ ont $t_{\mathrm{i}}$ disent que ... 'ceux qui ont vécu à Oslo disent que ...'

4) Alors que l'antéposition V2 peut se faire à longue distance (7a) (Thráinsson 2007: 351 ; voir aussi (8b)), l'antéposition stylistique est limitée, bornée, à la proposition-source (7b) (Ott 2009 : 145, ex. (13)) :

(7) a. Haroldi-Dat $t_{\mathrm{i}}$ heldur María [ að Jón treisti $t_{\mathrm{i}}$ ]

Harold-Dat croit Marie que Jean a-confiance-en

'Marie croit que Jean a confiance en Harold.'

b. * * Bókin ${ }_{\mathrm{k}}\left[\mathrm{CP}\right.$ sem stolið ${ }_{\mathrm{i}}$ var sagt [CP að pú hefðir $\left.t_{\mathrm{i}} t_{\mathrm{k}}\right]$

Le livre qui volé était dit que tu avais...

'Le livre qu'il était dit que tu avais volé ...' (= dont on disait que tu l'avais volé)

5) Alors que l'antéposition V2 d'un constituant dans une phrase subordonnée (d'un type permettant V2) bloque le déplacement à longue distance d'un autre constituant par-dessus lui (8a) (par antéposition V2 ou mouvement WH), un constituant antéposé stylistiquement ne le fait pas (8b) (les exemples en (8) sont de Ott [2009 : 145, exemples 14a-b]; cf. aussi Maling 1990, Rögnvaldsson and Thráinsson 1990, Jónsson 1991, Holmberg 2006) :

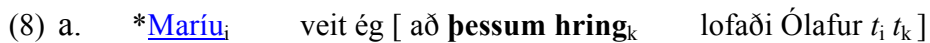

Maria-DAT sais moi que cette bague.ACC promit Olaf.Nom

'Je sais que Olaf a promis cette bague à Marie.'

b. pennan $\operatorname{mann}_{i}$ hélt ég [ að fario $x_{\mathrm{k}}$ hefði verið með $t_{\mathrm{k}} t_{\mathrm{i}}$ á sjúkrahús]

Cet homme pense moi que allé a été à hôpital

'Cet homme, j'ai pensé qu' (il) est parti à l' hôpital.'

6) l'antéposition stylistique est sujette à une importante contrainte d'accessibilité à laquelle l'expression déplacée par antéposition V2 est insensible : la négation ekki et les adverbes de phrase, plus généralement des adverbes situés haut dans la structure, ont préséance sur les participes et les particules verbales, qui ont eux-même préséance sur les adjectifs prédicatifs dans la formulation de Ott (2009: 152) ${ }^{4}$.

(9) Hiérarchie d'accessibilité

\{négation/adverbes \}»\{ participe passé/particule verbale \}»\{ adjectifs prédicatifs \}

7) l'antéposition stylistique requiert que le sujet n'occupe pas sa position canonique (SpecIP ou SpecTP). Autrement dit, l'absence du sujet de cette position est un préalable à l'antéposition stylistique (voir plus bas pour des exemples).

Ces deux dernières exigences sont les plus frappantes. Pour d'autres, on peut se référer à Ott (2009).

\subsection{Le déplacement stylistique à gauche (DSG) en ancien français}

Le déplacement stylistique à gauche de l'ancien français rappelle l'antéposition stylistique de l'islandais, mais s'en distingue de diverses manières. Considérons quelques points communs : les deux types de déplacement sont permis dans les relatives sujets, constructions qui ne permettent pas V2 ; le mouvement à longue distance (par topicalisation ou déplacement WH d'un relatif ou d'un interrogatif) est possible dans les deux langues hors de propositions où il y a eu antéposition/déplacement stylistique (cf. (8b) pour l'islandais et $(10 \mathrm{a}, \mathrm{b})$ pour le français).

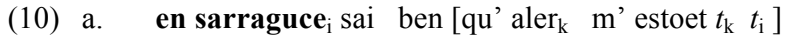

'Je sais bien qu'il me faut aller à Sarragoce'

[ROLAND, 23.283, MCVF]5
} 
b. Samuel cumanda que l'um aseïst devant Saül le mes real [que $\mathbf{q}_{\mathrm{i}}$ il out par purveance cumande [que á Saül $l_{\mathrm{k}}$ fust estúe $t_{\mathrm{k}} t_{\mathrm{i}}$ ]

'Samuel commanda que l'on dépose devant Saul le repas royal qu'il avait à l'avance commandé qui soit réservé à Saul.'

[QUATRELIVRE, 18.599, MCVF]

À l'inverse, l'antéposition stylistique à longue distance est exclue en islandais (ex. 7), et nous n'avons connaissance d'aucun cas de déplacement stylistique à gauche à longue distance en français.

Ces points communs entre l'antéposition stylistique de l'islandais et le déplacement stylistique à gauche de l'ancien français peuvent amener à penser que l'on se trouve en face du même phénomène grammatical. Toutefois, d'autres propriétés font ressortir des différences importantes. Dans les prochaines sections, nous nous attachons à montrer que la hiérarchie d'accessibilité de l'islandais (point 6) ne contraint pas le déplacement stylistique à gauche, que la contrainte du sujet (point 7) est sans effet en ancien français, et enfin, que les constituants lourds (point 3), notamment des VP, peuvent être antéposés en ancien français.

\subsection{Contrainte d'accessibilité (effets d'intervention)}

\subsubsection{Négation}

La généralisation descriptive exprimée par la contrainte d'accessibilité en (9) résulte, selon Holmberg (2006, section 5) d'un principe de localité, la Condition du Lien Minimal introduite dans Chomsky (1994) : s'il y a plusieurs candidats à l'antéposition stylistique, seul le candidat structuralement le plus proche de la position visée par l'antéposition stylistique, autrement dit, celui le plus haut dans la structure, peut être déplacé ; si deux candidats sont équidistants de la cible, ce qui est le cas s'ils sont structuralement des sœurs, n’importe lequel des deux peut se déplacer.

L'effet de la négation est illustré ci-dessous. Alors qu'un participe (11b) et un PP (11c) peuvent être antéposés dans une phrase non négative (Holmberg 2006 : exemples 12a, et 26a-b), leur antéposition n'est pas possible en (12c-d), où seul l'adverbe de négation peut s'antéposer (Holmberg 2006: exemples 39a-d).

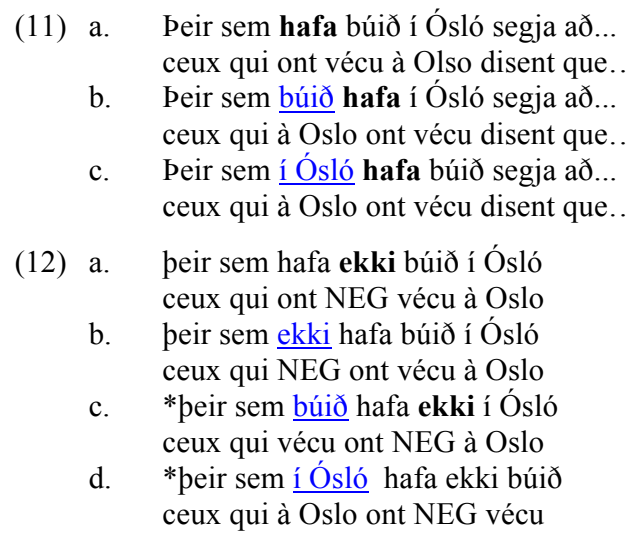

L'ancien français ne semble pas soumis à cette hiérarchie d'accessibilité. Ainsi, alors qu'un adverbe négatif comme pas/point/mie/plus peut être antéposé (cf. (13)), sa présence dans la phrase ne bloque pas l'antéposition d'un autre élément situé structuralement plus bas que lui (cf. (14)-(17)). ${ }^{6}$

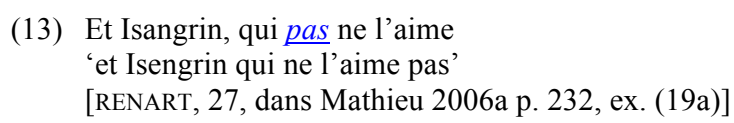


(14) Quant resembler nes poeit mie/ Par chasteé, par nette vie,/ Par servise volt purchacier/ Lur amur sanz rien losenger.

'Quand elle ne peut leur ressembler, elle veut obtenir leur amour sans les flatter en rien, par sa chasteté, sa vie pure, et son mérite.'

[ADGAR, 3e t., 12e s., v. 77, BFM] ${ }^{7}$

(15) Kant atenir ne se pout plus, / Chaïr se lait a terre jus ;

'Quand elle ne peut plus se soutenir, elle se laisse tomber à terre.'

[EUSTACE-PETERSEN, 60.817, MCVF]

(16) Huelin dist une noevele / qui a Gorm[un]d ne fut pas bele 'Huelin dit une nouvelle qui ne plut pas à Gormond.' [GORMONT, 239-240]

(17) Ja seit iceo que veirs n'est mie/ Que cist n'amast unkes folie, / Que nus quidum e saint e ber,

'Je sais bien qu'il n'est pas vrai que n'aima jamais la folie celui que nous pensons être saint et noble...'

[ADGAR, 3e t., 12e s., v. 61, BFM]

En (14), le forclusif mie est associé au verbe principal, alors que l'infinitif antéposé fait partie du VP complément de ce verbe. Si l'on se trouvait en face d'un cas d'antéposition stylistique, mie devrait être plus accessible que l'infinitif, puisqu'il occupe une position structuralement supérieure. Il en est de même pour plus en (15). Les exemples (16) et (17) illustrent l'antéposition d'un complément ( $a$ Gorm $[u] n t)$ et d'un adjectif prédicatif (veirs) par-dessus un forclusif. Si, suivant Pollock (1989) et d'autres, les forclusifs sont générés dans une position externe au VP, par exemple NegP, et que le verbe fléchi se déplace dans une position flexionnelle supérieure au forclusif, comme illustré en (18), alors le forclusif en (16) et (17) est structuralement supérieur à l'élément antéposé tout comme il l'est en (14) et (15), et on s'attendrait à ce qu'il bloque le déplacement stylistique si celui-ci était sujet à une contrainte d'accessibilité du type de celle observée en islandais.

$$
\text { (18) }\left[\mathrm{TP} \ldots \mathrm{n}^{\prime}+\mathrm{est}_{\mathrm{i}}\left[\mathrm { NegP } \text { mie } \left[\mathrm{vP} t_{\mathrm{i}}\right.\right. \text { veirs...]]] }\right.
$$

Étant donné qu'en islandais, ekki bloque l'antéposition stylistique de toute expression située plus bas, qu'il s'agisse d'une tête ou d'un syntagme (cf. les exemples 12c-d dans le texte), si (14)-(17) représentent des cas d'antéposition stylistique, il faudrait pouvoir expliquer pourquoi les éléments négatifs comme pas, point, mie ne bloquent l'antéposition stylistique d'aucun type d'expression qu'ils c-commandent asymmétriquement.

De manière générale, rien ne suggère qu'un adverbe intervenant entre la cible de l'antéposition stylistique, c'est-à-dire la position où l'élément déplacé doit aboutir, et l'expression à déplacer bloque l'antéposition de celui-ci en ancien français, contrairement à ce qui est le cas en islandais, notamment pour les adverbes de phrase ou plus généralement pour ceux situés dans une position structurale haute (cf. Holmberg 2006 : ex. (23)). Par exemple, le syntagme adverbial en (19) ne bloque pas l'antéposition du participe.

(19) Chadwalein ot grant marrement / Que returné fud tant suvent $t_{i}$; 'Chadwalein eut grande douleur / d'être si souvent retourné ;' [BRUT2, p. 742 v. 14176, BFM]

Il y a cependant très peu de subordonnées avec un adverbe qui soit clairement d'un type approprié dans notre corpus, et ceci mérite une recherche plus approfondie.

\subsubsection{Autres effets d'intervention}

D'autres types d'effet d'intervention discutés par Holmberg (2006, ex. 26c,d) ne sont pas observés en ancien français. Ainsi, en islandais, lorsqu'une proposition contient un participe et un prédicat second, seul le participe peut être antéposé (20), tandis qu'en ancien français, un attribut (du sujet ou de l'objet) dans une prédication seconde peut être antéposé (21). 
(20) a. maðurinn sem talinn ${ }_{i}$ er $t_{\mathrm{i}}$ rikur

l'homme qui considéré est riche

'l'homme qui est considéré riche' (Holmberg 2006, 25b)

b. *maðurinn sem rikur ${ }_{\mathrm{i}}$ er talinn $t_{\mathrm{i}}$

l'homme qui riche est considéré

'l'homme qui est considéré riche' (Holmberg 2006, 25c)

(21) a. Luy mort, sa gent fut desconfite et la cité prinse qui chier ${ }_{i}$ est vendue $t_{\mathrm{i}}$. [PERCEFOREST, 126.1180, MCVF]

b. Quant $\underline{v i v e}_{i}$ ad trovee s' amie $t_{\mathrm{i}}$, Ducement sa femme mercie.

[MARIE-DE-FRANCE, 189.3883, MCVF]

Enfin, alors qu'en islandais une tête et son complément sont également accessibles à l'antéposition stylistique, s'agissant de soeurs, l'antéposition d'un complément non sélectionné est impossible en présence d'un participe, comme on le voit en (22b), où le complément de lieu non sélectionné ne peut pas être antéposé par-dessus le participe passé.

$$
\begin{aligned}
& \text { (22) a. peir sem hitt hafa konuna sina í Ósló } \\
& \text { ceux qui rencontré AUX femme leur à Oslo } \\
& \text { 'Ceux qui ont rencontré leur femme à Oslo' } \\
& \text { b. } \quad \text { peir sem í Ósló }{ }_{\mathrm{i}} \text { hafa hitt konuna sina } t_{\mathrm{i}} \\
& \text { ceux qui à Oslo AUX rencontré femme leur }
\end{aligned}
$$

Avant de contraster ce type d'exemple à des exemples d'antéposition d'ajout en ancien français, notons que l'agrammaticalité d'exemples comme (22b) ne suit pas directement de ce qui a été dit jusqu'ici. En effet, traditionnellement, un complément non sélectionné, un ajout, est situé plus haut dans la structure que le participe, qui ne le c-commande pas, de sorte que le participe ne devrait pas être sur le parcours de l'élément antéposé (Ott 2009 : 161, note 31). Il est toutefois possible que même s'il n'est pas sélectionné par le verbe, ce type de locatif doive être traité structuralement comme complément du verbe, plutôt que comme un véritable ajout. Quoi qu'il en soit, en ancien français, un complément non sélectionné peut parfaitement être déplacé par-dessus un participe. ${ }^{8}$

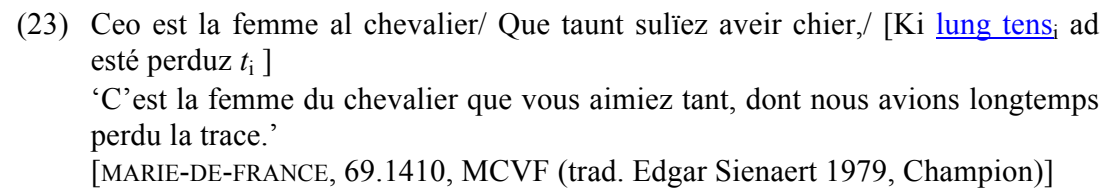

On en conclut que le type de déplacement à gauche observé en ancien français n'est soumis à aucune sorte de contrainte d'accessibilité, contrairement à ce qui est le cas de l'antéposition stylistique de l'islandais, ce qui suggère qu'il s'agit d'un processus grammaticalement distinct de celui de l'islandais.

\subsection{La condition du sujet}

Dans les propositions de type V2, l'ordre XP-verbe-sujet consécutif au déplacement du verbe à gauche du sujet et à l'antéposition V2 d'un élément en position initiale est licite quel que soit le type de sujet, incluant des pronoms personnels et des sujets définis, ce qui s'explique bien si l'élément initial et le verbe occupent la périphérie gauche de la phrase et le sujet sa position canonique (SpecTP), comme dans la représentation en (24) (Holmberg 2013).

$$
\text { (24) }\left[\mathrm{XP}_{\mathrm{k}}\left[\mathrm{FinP} \mathrm{V}_{\mathrm{i}}\left[\mathrm{TP} \text { sujet } t_{\mathrm{i}}\left[\mathrm{vP} t_{\mathrm{k}} \ldots . .\right]\right]\right]\right]
$$

À l'inverse, un préalable à l'antéposition stylistique est que le sujet n'occupe pas sa position canonique : il doit avoir été extrait par relativisation ou interrogation (comme on l'a vu en 11c), être nul (implicite) dans une construction impersonnelle (25), ou encore postverbal comme dans le passif impersonnel (26), auquel cas il est analysé comme faisant partie du VP. Dans ce dernier cas, le sujet doit être indéfini, les sujet nominaux définis et pronominaux étant nécessairement réalisés dans SpecTP. 
(25) Рað var hætt að rigna pegar komið $\underline{\mathrm{i}}_{\mathrm{i}}$ var $t_{\mathrm{i}}$ pangað (Maling, 1990 : 78, ex. 16a )

il était arrêté de pleuvoir quand arrivé fut là

'Il avait cessé de pleuvoir quand ils/nous sont/sommes arrivés là.'

(26) Allir vissu að stolið $\Varangle_{i}$ hafpi verið $t_{\mathrm{i}}$ smjöri (Hrafnbjargarson, $2004:$ ex.12) tous savaient que volé a été beurre

'Tous savaient que du beurre avait été volé.'

La condition du sujet associée à l'antéposition stylistique de l'islandais est illustrée en (27) et (28) :

(27) * рað var hætt að rigna pegar komin var rútan pangað

il était arrêté de pleuvoir quand arrivé fut autobus-déf. là

'Il avait cessé de pleuvoir quand l'autobus est arrivé là' (Maling, 1990 : 78, ex. $16 \mathrm{~b})$

(28) a. vinnan sem hann hefur haft í Ósló

emploi-déf. que il a eu à Oslo

'Le poste qu'il a eu à Oslo'

b. *vinnan sem hann í Ósló hefur haft

c. *vinnan sem í Ósló hann hefur haft

d. *vinnan sem í Ósló hefur hann haft

L'exemple (27), qui contraste avec (25), montre que l'antéposition stylistique est exclue avec un sujet nominal défini postverbal. Les exemples en (28), pris à Holmberg (2006 : ex. 12-14), montrent que l'antéposition stylistique est incompatible avec un sujet préverbal non extrait de IP et avec un sujet pronominal postverbal. Le cas ordinaire où le sujet et le complément locatif sont dans leur position canonique est illustré en (28a). Les exemples $(28 \mathrm{~b}, \mathrm{c})$ montrent que la cooccurrence à la gauche du verbe d'un sujet et d'un élément antéposé stylistiquement est impossible, et (28d) illustre l'impossiblilité de laisser le pronom en position postverbale (Hrafnbjargarson, 2004).

Mentionnons ici, puisque le fait est pertinent pour la comparaison avec l'ancien français, la possibilité, controversée, d'une exception à la condition du sujet en l'islandais. Hrafnbjargarson (2004: 117, ex. 79ab) accepte marginalement l'antéposition du participe (ou en général d'une tête, mais pas d'un XP) en présence d'un sujet préverbal, mais uniquement lorsque celui-ci est un pronom faible, comme 'ann (29a), à contraster avec la version forte de ce pronom, hann, en (29b) :

$$
\begin{aligned}
& \text { (29) a. ?Allt sem 'ann lesið } \varlimsup_{i} \text { hafði } t_{\mathrm{i}} \text { i bókinni war satt } \\
& \text { tout que il lu avait dans livre-le était vrai } \\
& \text { 'Tout ce qu'il avait lu dans le livre était vrai.' } \\
& \text { b. *?Allt sem hann } \underline{\text { lesið }}_{\mathrm{i}} \text { hafði } t_{\mathrm{i}} \mathrm{i} \text { bókinni war satt }
\end{aligned}
$$

Toutefois, Sigurðsson (2010 : 177, note 27) rejette les exemples comme (29a), et il souligne qu'il en est de même pour les autres linguistes islandais qu'il a consultés et qu'il n'a jamais observé d'exemple attesté de cette construction.

Nous pouvons maintenant aborder la question de savoir si le déplacement à gauche de l'ancien français qui nous concerne requiert que la position canonique du sujet ne soit pas occupée par celui-ci. La question est non seulement importante pour la description et l'analyse du phénomène lui-même, mais aussi pour sa disparition, puisque, selon Mathieu (2006a : section 4.3), cette antéposition aurait disparu lorsque les sujets nuls n'ont plus été possibles et qu'il relie les deux phénomènes (notre opinion est que ce n'est pas le cas, mais nous reportons la discussion de ceci à une autre occasion, faute d'espace). Pour y répondre, nous avons utilisé le corpus MCFV d'ancien et moyen français (Martineau et coll. 2010), corpus de textes analysés syntaxiquement auquel nous avons ajouté des textes traités au laboratoire d'Anthony Kroch de l'université de Pennsylvania), et nous y avons ciblé des éléments qui caractérisent l'antéposition stylistique en islandais. Nous avons donc étudié l'antéposition en subordonnées de verbes non fléchis (infinitifs et participes passé) et de forclusifs et autres petits adverbes dont on s'attend à ce qu'ils occupent une position élevée dans la phrase (pas, point, molt, mie, mult, mut, bien, ben, tant, trop). Comme le montrent les résultats du tableau 1, la condition du sujet vide n'est pas un prérequis au déplacement stylistique typique de l'ancien français. Si l'antéposition se fait la plupart du temps avec des 
sujets nuls en subordonnée (colonne 1), et rarement avec des sujets postverbaux (colonne 2), l'antéposition en présence d'un sujet préverbal est toutefois observée dans plus d'un cas sur dix $(13 \%)$ (colonnes 3-4-5). On a donc ici une autre différence, importante, avec l'islandais.

Tableau 1

\begin{tabular}{|c|c|c|c|c|c|}
\hline & \multicolumn{5}{|c|}{ Subordonnées } \\
\hline & 1 & 2 & 3 & 4 & 5 \\
\hline & X V Ø & X V S & X S V & S X V & $\%$ (col. 3+4) \\
\hline Infinitifs & 437 & 6 & 10 & 72 & $16 \%$ \\
\hline Participes & 537 & 42 & 18 & 71 & $13 \%$ \\
\hline Adverbes & 624 & 30 & 41 & 35 & $11 \%$ \\
\hline & $83 \%$ & $4 \%$ & $4 \%$ & $9 \%$ & \\
\hline
\end{tabular}

Mathieu (2006a, 2013), qui insiste sur les ressemblances entre l'ancien français et l'islandais, développe une analyse de l'ancienne langue qui permet de dériver l'ordre de mots XSV lorsque le sujet est nominal (DP) et l'élément déplacé une tête. L'ordre obtenu est donc l'opposé de celui de l'exemple islandais controversé (29a) présenté par Hrafnbjargarson, où le sujet devrait être un pronom faible et précéder le constituant antéposé. Dans l'analyse de Mathieu, un XP antéposé stylistiquement doit passer par la position sujet, utilisée comme "tremplin" (Mathieu 2007) pour sortir de la phrase et atterrir en SpecTop ${ }^{+} \mathrm{P}$, une catégorie située dans la périphérie gauche de la phrase. Le déplacement est évidemment impossible si un sujet occupe la position “tremplin". Par contre, le déplacement d'une tête n'est pas bloqué par un sujet plein dans SpecTP (une position de XP), ce qui permet, par exemple, à un participe de se déplacer dans la périphérie gauche. ${ }^{9}$

\section{(30) $[$ HangTopic/Left dislocation $[$ TopP ... [Top+P__ [FocusP $[$ FinP $[$ TP ...]]]]}

Mathieu met donc de l'avant l'existence d'un ordre XSV limité aux cas où X est une tête et S un DP plein. Toutefois, les données du corpus MCVF, résumée dans le tableau 1 ci-dessus, montrent que, dans le cas des participes et des infinitifs, l'ordre SXV est en fait beaucoup plus fréquent que l'ordre XSV (c'est moins le cas lorsque $X$ est petit adverbe). Le tableau 2, qui sépare les données en périodes de 50 ans, montre également une évolution dans l'ordre des mots. En ancien français, l'ordre SXV domine largement, mais à compter du début du $14^{\mathrm{e}} \mathrm{s}$. on note un renversement de tendance vers l'ordre XSV. Une étude plus approfondie serait nécessaire pour déterminer les causes de ce changement.

Tableau 2

\begin{tabular}{|c|c|c|c|}
\hline & & \multicolumn{2}{|c|}{ Subordonnées } \\
\hline & & XSV & SXV \\
\hline Ancien français & $<1150$ & 0 & 10 \\
\hline & $1150-1199$ & 0 & 98 \\
\hline & $1200-1249$ & 2 & 15 \\
\hline & $1250-1299$ & 1 & 22 \\
\hline & $1300-1349$ & 1 & 0 \\
\hline Moyen français & $1350-1399$ & 50 & 20 \\
\hline & $1400-1449$ & 0 & 0 \\
\hline & $1450-1499$ & 12 & 0 \\
\hline XVI $^{\mathrm{e}}$ s. & & 3 & $\mathbf{1 7 8}$ \\
\hline & Totaux & $\mathbf{6 9}$ & \\
\hline
\end{tabular}

Considérons d'abord l'ordre SXV. Cet ordre est très régulièrement observé dès l'ancien français tant avec les sujets nominaux que pronominaux, et autant avec des antépositions de têtes (31) qu'avec des constituants plus larges (32). Cette construction est rejetée par la plupart des linguistes islandais, et, bien 
que Hrafnbjargarson accepte marginalement l'ordre pronom faible - participe - verbe pour l'islandais, il rejette l'ordre de mots où l'élément antéposé n'est pas un participe mais un constituant plus large et où le sujet n'est pas pronominal (Hrafnbjargarson, p. 118).

(31) a. Eüstaces mot n'en savoit / De ço que Dex sauvé avoit ses effanz...

'Eustache ne savait mot de ce que Dieu avait sauvé ses enfants...'

[EUSTACE-FISHER, 31.370, MCVF]

b. por savoir s'il verroit riens de la chose qu'il plus desirroit .

'pour savoir s'il verrait quoi que ce soit de la chose qu'il désirait le plus.' [QUESTE, 82.2217, MCVF]

(32) a. Voirs est que je nul enfant ne puis avoir de vous

'il est vrai que je ne puis avoir aucun enfant de vous'

[CASSIDORUS, 136.884, MCVF]

b. Et leur di, s'il bien creü unt Dieu le Pere de tout le munt/...

'et il leur dit (que) s'ils ont bien cru en Dieu, le Père de tout le monde ...' [BORON, 88.1396, MCVF]

c. - Et qui sont cil qui tant vos ainment don li uns si hardiz seroit qu'a trois conbatre s'oseroit por vos sauver et garentir?

'et qui sont ceux qui vous aiment tant, dont un seul serait si hardi qu'il oserait combattre contre trois pour vous sauver et vous protéger?'

[YVAIN, 110.3846, MCVF]

d. Si vil estoit et si puanz / Que nus o lui n'iert habitanz.

'il était si vil/sale et si puant que nul n'habitait avec lui.'

[BORON, 35.545, MCVF]

Cet ordre SXV est également attesté dans d'autres langues romanes médiévales ainsi qu'en italien contemporain, comme on peut le voir ci-dessous. Pour l'ancien portugais, on trouve l'exemple (33) chez Martins (2011), qui observe que l'ordre SXV est plus commun que l'ordre XSV dans cette langue ; pour l'ancien espagnol, Rivero (1992) donne (34), parmi d'autres, et pour l'ancien catalan, Batllori et coll. (2005) présentent (35). Il faut remarquer qu'en (34) et (35), la négation ne bloque pas le déplacement à gauche. Pour l'italien moderne, Cardinaletti (2003) montre également que l'élément antéposé peut suivre un sujet préverbal (36), et on remarque que la négation ne bloque pas le déplacement.

(33) de quem.quer que uos algũu enbargo sobrel quiser põer

de quiconque que vous un quelconque obstacle sur-ce veut mettre

'[vous protégeant] de quiconque essaie de vous l'interdire'

[Document légal, 1381. Cf. Martins 2001 : 459; Martins 2011 : 146, (22a)]

(34) E pues yo gradescer non vos lo puedo

Et puisque je remercier neg vous le puis

'Et puisque je ne peux pas vous remercier pour cela.'

[Libro del Caballero Zifar, éd. J. González Muela, Madrid, Castalia, 1982, 1.362, in Rivero $1992: 265$.]

(35) E que aviem guarit que nos dar non.o deviem

Et que nous-avions considéré que nous donner pas le devions

'et que nous avions considéré que nous n'avions pas à le donner'

[XIII. Russell-Gebbett 1965 : 109 ; Lettre à l'évêque d'Urgell (1257-1269), in

Batllori et coll. 2005 (70a)]

(36) La ragione per la quale si può dire che il problema risolto non è

'la raison pour laquelle on peut dire que le problème n'est pas résolu'

[Cardinaletti, 2003, ex. 9]

L'ordre de mots SXV n'est pas exclusif des langues romanes. Ainsi, Hrafnbjargarson (2004 : ex. 74) signale l'existence de cet ordre en moyen danois (37) et Fischer (2014: ex. 36) cite un exemple d'Árnason (2002 : 205) en proposition principale en ancien islandais (38). 
(37) som han sidhan hafdhe fongit

'que il alors avait reçu'

[Sjoelens Trøst 1325]

(38) Hlaðguðr ok Hervör borin war Hlöðvé(i).

Hlaðguðr et Hervör nés étaient Hlöðvér.DAT

'Hlaðguðr et Hervör étaient nés à Hlöðvér.'

[Völundarkviða 15, 1-2]

Ces exemples soulèvent la question de savoir si cette construction en ancien scandinave témoigne de l'antéposition stylistique sans la contrainte du sujet, ou s'il s'agit d'une autre construction, pareille à celle de l'ancien français. Dans ce dernier cas, l'antéposition stylistique de l'islandais moderne pourrait être un résidu de cette construction plus ancienne, limité aux cas où le sujet n'occupe pas SpecTP. Il faudrait toutefois une plus large documentation permettant de vérifier si ce type d'exemple est sujet ou non aux contraintes qui restreignent l'application de l'antéposition stylistique.

Comme nous l'avons dit, contrairement à l'ordre SXV, l'ordre XSV est pratiquement inexistant en ancien français. Les deux exemples du tableau 2 à la ligne 1200-1249 se trouvent dans Clari (1205) et impliquent tous deux l'expression com fais.

(39) Or vous dirai du moustier Sainte Souphie com fais il estoit;

'Or je vous dirai, au sujet du moustier Sainte Sophie, comment il était fait ;'

[CLARI, 85.2107, autre exemple de 'com fais il estoit' 85.2118. MCVF]

L'existence du mot comfaitement 'comment' (com fait + ment) permet de penser que (39) illustre l'antéposition d'un constituant interrogatif complexe com fais, plutôt que l'antéposition indépendante de fais.

Si l'on met donc de côté ces deux exemples, le premier exemple de la construction XSV dans notre corpus date de la deuxième moitié du $13^{\mathrm{e}} \mathrm{s}$. (Cassidorus, vers 1270). Nous avons observé cet ordre de mots pratiquement exclusivement avec des sujets pronominaux, comme en (40)-(41), bien qu'il y ait quelques exceptions, comme les deux que l'on peut voir en (42) (on notera, dans ce dernier exemple, l'alternance de position: 'bien li nostre $\mathrm{V}$ ' versus 'Li autre mieus $\mathrm{V}$ ', qui suggère que le choix est vraiment stylistique ici).

(40) Dont dist cilz que voirs est que parlé on en avoit, et que bon seroit qu'ele s'en gardast.

'Alors celui-ci dit qu'il était vrai que l'on en avoit parlé et qu'il serait bon qu'elle fasse attention.' [CASSIDORUS, 138.923, MCVF]

(41) Et trop grant temps avoit que point il ne s'estoient veu en parti de bataille avoir 'et il y avait trop longtemps qu'il ne s'étaient pas vu en situation d'avoir une bataille'

[FROISSART, 724.15707, MCVF]

(42) a. Il feïrent une ordenance/ Qu'esleüs xij. homes seroient/ Qui le païs gouverneroient,

'ils firent une ordonnance selon laquelle seraient élus 12 hommes qui gouverneraient le pays' [PRISE, .4632, MCVF]

b. Et se bien li nostre assailloient,/ Li autre mieus se deffendoient. 'et si les nôtres assaillaient bien, les autres se défendaient mieux' [PRISE, .1453, MCVF]

Pour conclure, nous avons vu que contrairement à l'antéposition stylistique de l'islandais, la présence de sujets préverbaux ne fait pas obstacle au déplacement stylistique à gauche en ancien français, et que l'ordre non marqué pour le déplacement stylistique est celui où le sujet précède l'élément antéposé. L'antéposition caractéristique de l'ancien français, le déplacement stylistique à gauche, est donc distincte de l'antéposition stylistique de l'islandais. Les exemples (33) à (36) montrent que les autres langues 
romanes sont plus proches de l'ancien français que de l'islandais, de sorte qu'il faudrait dans ces cas-là aussi sans doute parler de déplacement stylistique à gauche plutôt que d'antéposition stylistique. Il reste à voir si outre des exemples du type de (37)-(38) de périodes anciennes des langues scandinaves, ces états de langues contiennent également des cas d'antéposition qui violent les autres caractéristique de l'antéposition stylistique, une question qui ne peut être résolue qu'à partir d'études futures des spécialistes de ces langues.

\subsection{Deux constituants à gauche du verbe}

Holmberg (2006, section 2.3, p. 7) décrit l'antéposition stylistique comme ne pouvant déplacer qu'une seule catégorie par proposition. Mais Hrafnbjargarson (2004) soutient qu'il peut y avoir double antéposition. Un premier type d'exemple est celui controversé d'un pronom sujet faible suivi d'une tête (voir (29a)). Mais Hrafnbjargarson (2004 : 121-129, ex. 94 et 103) mentionne également l'antéposition de deux adverbes en islandais, dont l'un est la négation, ou d'un adverbe suivi d'un participe.

(43) Hann henti öllu sem áreiðanlega ekki hafði verið taemt il jeta tout qui indubitablement pas avait été vidé 'Il jeta tout ce qui n'avait sans aucun doute pas été vidé.'

(44) Nemendur sem ádur lokið hafa sambaerilegum námsaföngum étudiants qui avant fini avaient semblables cours 'Des étudiants qui avaient terminé des cours semblables auparavant.'

Pour cet auteur, la catégorie ciblée par l'antéposition stylistique est $\mathrm{Foc}(\mathrm{P})$ dans la périphérie gauche de la phrase (avec la hiérarchie TopP $>$ FocP $>$ IP). La double antéposition est le résultat du déplacement de la première expression comme un XP dans la position de spécificateur de SpecFocus, et du déplacement de la seconde dans la position de tête de cette même projection. Dans (43) on peut intervertir l'adverbe d'opinion et la négation. Pour Hrafnbjargarson, cela signifie que ces deux éléments ont un double statut : ils peuvent fonctionner comme des XP ou comme des têtes (l'ordre de surface reflète l'ordre relatif avant les déplacements). Par ailleurs, Wood (2011 : note 19) précise qu'une double antéposition est exclue en islandais dans certains cas, par exemple, il ne serait pas possible d'antéposer à la fois un verbe et son complément.

Considérons maintenant le français. Mathieu (2006a, 2006b, 2013) argumente que deux éléments peuvent être antéposés stylistiquement en ancien français, et il présente une analyse qui partage l'aspect central de celle de Hrafnbjargarsson, à savoir que les éléments antéposés se déplacent dans la périphérie gauche de la phrase, dans le spécifieur ou la tête d'une catégorie fonctionnelle, la différence concernant l'identité de cette catégorie, étiquetée Top ${ }^{+} \mathrm{P}$ par Mathieu. Cette analyse, tout comme celle de Hrafnbjargarson, prédit qu'on ne peut trouver que l'ordre $\mathrm{XP}-\mathrm{X}^{0}$ dans le cas de double antéposition stylistique. C'est effectivement cet ordre qu'on trouve dans l'exemple (45) de Mathieu :

(45) Se lieve sus, et cil le voient /Qui avoec lui aler devoient

'il se lève et ceux qui devaient aller avec lui le voient'

[CHARRETTE, 2203-2205, in Mathieu 2006a ex. (24b)]

Toutefois, on remarquera que dans cet exemple, les deux éléments qui auraient été déplacés par antéposition stylistique sont le verbe et son complément (entendu au sens large, il ne s'agit pas nécessairement de complément sélectionné), ce qui est exclu en islandais, selon Wood (2011).

De plus, dans les textes que nous avons étudiés, on trouve diverses exceptions à la contrainte XP- $\mathrm{X}^{0}$. On peut trouver à la gauche du verbe conjugué divers ordres de surface, $\mathrm{X}^{0}-\mathrm{XP}, \mathrm{X}^{0}-\mathrm{X}^{0}$, et XP-XP, comme nous le détaillons ci-dessous. Ainsi, dans les exemples $(46 \mathrm{a}, \mathrm{b})$, le participe et son complément précèdent l'auxiliaire dans l'ordre $\mathrm{X}^{0}$-XP (en outre, dans (46a), le participe est précédé de l'adverbe 'bien' qui le modifie) :

(46) a. Einsi-comme apres le sarez,/ Quant [bien leü ce livre] arez.

'ainsi comme vous le saurez plus tard, quand vous aurez bien lu ce livre'

[PRISE, .135, MCVF] 
b. Vos savez bien que [desguarni / De touz avoirs] sonmes ichi 'vous savez bien que nous sommes ici dépouillés de tous avoirs' [EUSTACE-PETERSEN, 27.366, MCVF]

Dans ce type de cas, il est naturel de penser que plutôt que d'avoir des déplacements indépendants, c'est tout le VP qui a été déplacé stylistiquement à gauche (en fait, cela semble la seule chose possible dans un cas comme 46a). Ce type de déplacement est agrammatical en islandais pour l'antéposition stylistique (cf. ci-dessus, section 2.1, point 3), et Mathieu (2006a : 241) le considère comme exclu en ancien français, contrairement à ce qu'on voit en (46).

Par ailleurs, on trouve également des cas d'antéposition de deux têtes verbales, tant dans l'ordre V1-V2 (ex. 47a,b), où le verbe régisseur (V1) précède le verbe complément (V2), que dans l'ordre V2-V1 (48ab).

(47) a. Et s'en parti en moys de May, Si com $\underline{\text { aissié }}_{1} \underline{\text { dire }}_{2}$ le m' ay.

'et il me quitta au mois de mai, ainsi que je me le suis laissé dire.'

[PRISE, .812, MCVF]

b. Dusqu'a un jour [qu'alez ourer $_{2} /$ Fu Joseph devant sen veissel] ;

'Jusqu'à un jour où Joseph était allé prier devant sa coupe ;'

[BORON, 100.1595, MCVF] (v. 29734-5)

(48) a. Car vraiement dire pourra/ Que il $\underline{\operatorname{seisi}}_{2} \underline{v e u ̈}_{1}$ aura/ Hebron le Riche

Pescheeur/ Et dou veissel et de l'onneur

'car il pourra dire en toute vérité qu'il aura vu Hébron le Riche Pêcheur mis en possession et de la coupe et de l'honneur.' [BORON, 118.1844, MCVF]

b. Si com $\underline{\text { dire }}_{2}{ }_{2}$ oy 1 l'ai celi Qui y estoit

'Ainsi que je l'ai entendu dire (de) celui qui y était'

[PRISE, .1680, MCVF]

Dans (47a), le plus naturel est de dire que le VP maximal [VP1 $\mathrm{V}_{1}\left[\mathrm{VP}_{2} \mathrm{~V}_{2}\right]$ ] est antéposé ; dans (47b), il peut s'agir d'un cas de déplacement de VP résiduel (« devant son veissel » est d'abord extrait et le VP résiduel privé du DP extrait, [VP1 alez $_{1}$ [VP2 ourer $_{2}$ devant sen veisse1]], est ensuite déplacé stylistiquement à gauche). Pour une suite V2-V1 apparaissant à gauche du verbe tensé, comme en (48a), une possibilité serait qu'il y a eu un déplacement indépendant de chaque verbe, une autre que le DP 'Hebron le Riche Pescheeur' a été extrait de [vp1 veü [Hebron le Riche pescheeur [vP2 seisi]]], suivi du déplacement stylistique à gauche du VP résiduel [vp1 veü [Hebren le Riche pescheeur [vP2 seisi]]], l'ordre final étant obtenu par déplacement de $\mathrm{VP}_{2}$ soit dans la position de Spec de $\mathrm{VP}_{1}$ (ce qui donne une configuration de type $\mathrm{OV}$, soit par adjonction de $\mathrm{VP}_{2}$ à $\mathrm{VP}_{1}$. L'exemple (48b) serait dérivé de manière semblable.

Enfin, on trouve également à la gauche du verbe fléchi des ordre de mots de type $\mathrm{XP}_{2}-\mathrm{XP}_{1}$, qui pourraient résulter de l'antéposition du VP, accompagné de l'antéposition de l'objet direct à l'intérieur de celui-ci.

(49) Se trestout ce 2 bien gardé 1 unt

s'ils ont bien gardé tout cela

[BORON, 88.1396, MCVF]

On voit donc qu'on peut trouver à gauche du verbe fléchi une diversité d'éléments qui ne se limitent pas à ceux observés en islandais. 


\section{Analyse des ordres (S)XV et XSV}

Nous avons vu qu'en présence d'un sujet préverbal, l'élément ou les éléments antéposés se trouvent le plus souvent, en ancien français, entre le sujet et le verbe, alors qu'en moyen français, la construction la plus courante a l'ordre où le sujet suit l'élément antéposé. Dans cette section, nous discutons ces deux constructions. En ce qui concerne la périphérie gauche, nous adoptons la structure de Benincà et Poletto (2004) qui comporte les domaines illustrés en (50):

\section{(50) $[$ ForceP $[$ FrameP $[$ TopP $[$ FocusP $[$ FinP $[\ldots]]]]$}

Les complémenteurs et relatifs sont réalisés dans ForceP, les compléments scéniques initiaux dans FrameP, les éléments disloqués à gauche dans TopP et finalement, les éléments interrogatifs et les focus contrastifs sont dans FocusP. Dans les propositions V2, Fin ${ }^{0}$ reçoit le verbe.

\subsection{SXV et $ø X V$}

Parmi les cas de déplacement que nous examinons, la construction $\mathrm{SX}_{\mathrm{DSG}} \mathrm{V}$, où $\mathrm{X}_{\mathrm{DSG}}$ est l'élément déplacé stylistiquement à gauche, est celle dont l'analyse est, dans ses grandes lignes du moins, la plus claire, au sens où il n'y a pas de raison de penser que le sujet et le verbe fléchi y occupent des positions différentes de leur position canonique. Le constituant déplacé à gauche n'est donc pas dans la périphérie gauche de la phrase, mais plutot inséré entre le sujet et le verbe. On peut rendre compte de cet ordre de mots de manière simple en adoptant la position de Rizzi et Shlonsky $(2006,2007)$ pour qui la position canonique du sujet est celle de Specifieur d'une projection SubjP qui domine TP, le verbe fléchi occupant la tête de TP, comme en (51):

(51) [SubjectP [SpecSubject sujet [тр [т verbe fléchi] [vр … ] ] ]]]

L'élément déplacé stylistiquement pourrait alors se trouver dans une projection fonctionnelle facultative (que 1'on appellera DSGP), peut-être réitérable, insérée entre SubjP et TP, comme en (52).

(52) por savoir s'il verroit riens de la chose qu' [Subjp il [DSGP plus [тP desirroit ]]] 'pour savoir s'il verrait quoi que ce soit de la chose qu'il désirait le plus.' [QUESTE, 82.2217, MCVF et BFM]

Une représentation de type (52) semble préférable à l'analyse la plus courante, qui fait l'économie d'un niveau SubjectP et place le sujet dans la position de SpecTP ([TP [SpecTP sujet] [T, [T V. fléchi] [VP ... ]]]), puisqu'elle permet l'insertion entre le sujet et le verbe de l'élément déplacé à gauche sans postuler d'adjonction de celui-ci à T ou à T' et sans permettre la multiplication de spécifieurs.

Un argument indépendant à l'appui de l'idée que les éléments déplacés stylistiquement à gauche occupent des positions internes à la proposition minimale, c'est-à-dire la partie de la proposition n'incluant pas la périphérie gauche, c'est que les éléments de la périphérie gauche précèdent toujours le sujet, qui luimême précède l'élément déplacé déplacé stylistiquement à gauche. C'est notamment le cas dans les questions indirectes avec sujet préverbal (53a-b). Si le constituant interrogatif occupe la position Focus dans la périphérie gauche, on voit mal comment insérer à sa droite, mais dans la périphérie gauche, à la fois le sujet et l'élément déplacé stylistiquement.

(53) a. Dame, font il, pour Dieu nous dites/ [FocusP Ou [FinP [SubjP vous [FP $\underline{\text { cest }}$ suaire [тр preïstes]]]]]

'Dame, disent-ils, au nom de Dieu, dites nous où vous avez pris ce suaire.' [BORON, 55.847, MCVF] (v.1592-3, éd. Cerquiglini)

b. Ainz qu'il murget voldreit vetheir / Quel séd li bon devrunt aveir, / Quel lu li mal aveir devrunt 'avant de mourir, il voudrait voir quel siège les bons devont avoir, quel lieu les mauvais devront avoir' [BRENDAN, 31.26, MCVF] 
Pour les phrases d'ancien français où la position canonique du sujet est inoccupée (le sujet étant réalisé ailleurs ou nul) ou peut-être simplement absente (sans représentation structurale), nous adoptons le même type de structure, avec une projection DSGP par-dessus TP :

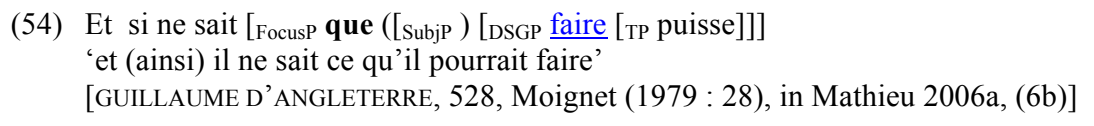

Un argument supplémentaire pour postuler que le constituant déplacé reste dans la proposition minimale est le fait que, si le constituant déplacé stylistiquement à gauche était déplacé dans la périphérie gauche, tout comme les termes relatifs et interrogatifs (comme dans les analyses de Mathieu pour l'ancien français et de Hrafnbjargarson pour l'islandais), dans les questions indirectes ci-dessus, il y aurait deux déplacements A-barre. Or, Poletto (2005), s'appuyant sur Haegeman (1997) et Roberts (1999), souligne que le déplacement A-barre de deux éléments dans la périphérie gauche devrait être exclu par la condition de minimalité, le deuxième intervenant dans la relation entre le premier et sa trace. Nous avons déjà vu que le déplacement stylistique à gauche n'interfère pas avec le déplacement $\mathrm{WH}$, que celui-ci soit à courte distance, comme en (52) à (54) ci-dessus, ou à longue distance, comme dans les exemples de (55), ce qui est attendu si le déplacement stylistique à gauche est confiné à la proposition minimale. (Il en est de même pour l'islandais, où l'antéposition stylistique n'interfère également pas avec le déplacement à longue distance, ce qui appuie les analyses où la cible de l'antéposition est interne à la proposition minimale [Holmberg 2000, 2006]).

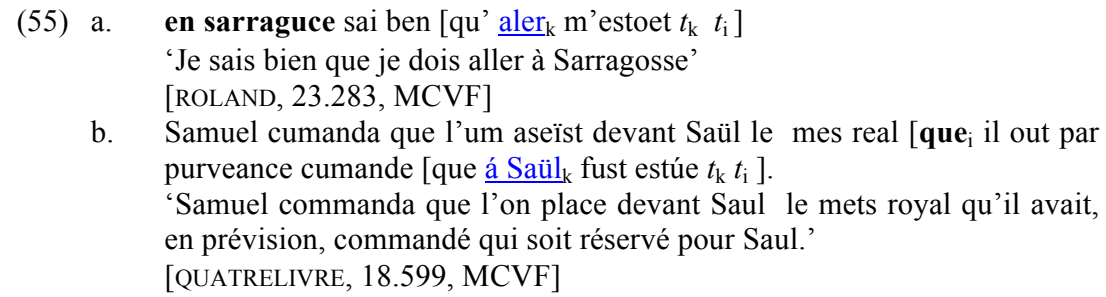

Finalement, si le qui des relatives sujet occupe Fin ${ }^{0}$ (Rizzi 1997) (56), ou si le -i de qui occupe la position canonique du sujet (Taraldsen, 2001) ou Fin ${ }^{0}$ (Rizzi \& Shlonsky, 2007), l'antéposition stylistique, qui est particulièrement favorisée par les relatives sujets, doit nécessairement cibler une position qui suit Fin ${ }^{0}$, situé à la frontière droite de la périphérie gauche, donc une position interne à la proposition minimale.

(56) come cil font [FinP qui $\mathbf{i}_{\mathrm{i}}\left[\operatorname{Subjp}_{\mathrm{i}} t_{\mathrm{i}}\left[\right.\right.$ [en queste $\left.{ }_{\mathrm{k}}\right]$ [TP doivent entrer $\left.\left.\left.\left.t_{\mathrm{k}}\right]\right]\right]\right]$

'comme font ceux qui doivent entrer dans une quête'

[QUESTE, §32, in Mathieu 2006a, ex. (1)]

En conclusion, les faits considérés dans cette section appuient l'idée que le déplacement stylistique à gauche a pour cible un domaine fonctionnel facultatif, interne à la proposition minimale, plutôt qu'une position appartenant à la périphérie gauche.

\section{$3.2 \mathrm{XSV}$}

Nous avons vu que l'ordre XSV est extrêmement rare en ancien français, (si l'on exclut les deux exemples avec com fais, il n'apparaît pas dans nos données avant la fin du $13^{\mathrm{e}} \mathrm{s}$.), mais devient plus fréquent en moyen français (cf. tableau 2).

Puisque dans les exemples de ce type l'élément déplacé semble généralement focalisé, mis en évidence, ce qui n'est pas le cas de l'élément déplacé des constructions vues jusqu'ici, une hypothèse possible est que cet élément occupe une position dans la périphérie gauche, peut-être une position de Focus, comme illustré en (57a). Alternativement, il pourrait être adjoint à la proposition minimale (SubjP — ou TP dans une approche plus traditionnelle), comme en (57b). Quelle que soit l'analyse, ce type de déplacement n'est pas un cas de la construction de déplacement stylistique à gauche discutée en 3.1, puisque la position ciblée est différente. 
(57) a. Et les clés avec li enporte,/ [Pour ce [ForceP qu' [FocusP entrer ${ }_{i}$ [Subjp on n’y peüst $t_{\mathrm{i}}$ ] ]]]

'Et il emporte avec lui les clés pour qu'on n'y puisse entrer ni sortir' [PRISE, .2738, MCVF]

b. Et vendoient les villes que pris avoient as honmes dou pais et a ceuls meismes [Forcep lesquels [subjp boutés hors [subjp il en avoient ]]]

'et il vendaient les villes qu'ils avaient pris aux hommes du pays et à ceux mêmes qu'ils en avaient chassé, ...'

[FROISSART, 858.18847, MCVF]

Étant donné ce que nous avons dit de l'impossibilité d'avoir deux mouvements A-barre dans la même proposition, l'analyse avec adjonction devrait être préférée, puisqu'en (57b) on a à la fois relativisation et déplacement à gauche. Alternativement, une distinction pourrait être faite entre deux types de mouvements dans la périphérie gauche. Une telle distinction est défendue par Frey (2006). Cet auteur distingue ce qu'il appelle 'true A-bar movement', qui peut se faire à longue distance et cible SpecFocP, et 'formal movement', qui est strictement local et cible Spec,FinP. Un corpus plus étendu est nécessaire pour déterminer l'ensemble des propriétés de ces constructions. Par exemple, (58) suggère que cette construction permet le déplacement à gauche non seulement d'un VP, mais aussi d'une projection contenant à la fois le forclusif et le VP.

(58) et furent les nefs recargiés de tout ce que il veoient que point mener il n'en pooient.

'et les bateaux furent rechargés de tout ce qu'ils voyaient qu'ils ne pouvaient pas amener.'

[FROISSART, 75.791, MCVF]

\section{Résumé et conclusion}

Dans ce travail, nous avons comparé le déplacement stylistique d'éléments à gauche du verbe tensé en ancien français à l'antéposition stylistique de l'islandais. Nous avons montré que les caractéristiques de l'antéposition stylistique de l'islandais ne se retrouvent pas en ancien français, ce qui nous a amené à considérer que les faits de l'ancien français devaient faire l'objet d'une analyse différente et se voir attribuer une autre désignation, et nous avons parlé ici de déplacement stylistique à gauche. Nous avons vu en particulier qu'il ne semblait pas y avoir de hiérarchie d'accessibilité en ancien français, ni de condition du sujet, et qu'on peut trouver à gauche du verbe fléchi une variété d'éléments, incluant des VP entiers ou résiduels. Dans la dernière section de l'article, nous avons examiné plus en détail deux types de constructions, celle de type (S)XV et celle de type XSV. En ce qui concerne la construction (S)XV, fréquente en ancien français, elle est le résultat du déplacement d'une tête et/ou d'un syntagme à la gauche immédiate de TP et à la droite de la position canonique du sujet (que celui-ci soit réalisé dans cette position, ou non). Pour la construction XSV, qui se développe à partir du dernier tiers du XIIIe siècle, nous avons considéré que l'antéposition ne relevait pas de la construction de déplacement stylistique à gauche donnant l'ordre SXV, et pourrait cibler la périphérie gauche ou créer une structure d'adjonction à la proposition minimale. Pour l'instant, le corpus d'exemples ne permet pas d'aller bien loin au-delà de ces hypothèses.

Nous concluons qu'il n'y a pas une seule construction avec déplacement stylistique, mais au moins deux dans les subordonnées, et qu'il y a un changement entre l'ancien français et le moyen français, l'ancien français privilégiant l'ordre SXV et le moyen français développant l'ordre XSV, un sujet qui a été laissé à une étude ultérieure.

\section{Références des exemples cités}

ADGAR (Adgar, dit Guillaume), Collection de miracles, éd. P. Kunstmann. Ottawa, Université d'Ottawa, 1982. Publié en ligne par la Base de français médiéval, http://catalog.bfm-corpus.org/adgar. Dernière révision le 2011-03-23.

BORON (c. 1190). Le roman de l'estoire dou Graal, éd. W. A. Nitze. Paris : Champion, 1927. 
BRENDAN (c.1120). The Anglo-Norman voyage of St Brendan, éd. I. Short \& B. Merrilees. Manchester : Manchester University Press, 1979.

BRUT2 (achevé en 1155). Wace, Le roman de Brut, éd par I. Arnold. Oxford : Anglo-Norman Text Society, 19381940. Publié en ligne par la Base de français médiéval, http://catalog.bfm-corpus.org/brut2. Dernière révision le 2011-11-07.

CASSidorus (c. 1270). Le roman de Cassidorus, éd. J. Palermo. Paris: Editions Picard, 1964.

Charrette (c. 1180). Chrétien de Troyes, Le Chevalier à la Charrette, éd. A. Foulet et K. D. Uitti. Publié en ligne par The Princeton Charrette Project http://www.princeton.edu/ lancelot/ss/materials.shtml (acc. le 27 fév. 2014).

ClARI (c. 1205). Robert de Clari, La Conquête de Constantinople, éd. Ph. Lauer. Paris : Champion, 1924.

COnStAntinOPLE (c. 1212). Geoffroy de Villehardouin, La conquête de Constantinople, éd. J. Dufournet. Paris : GF Flammarion, 2004.

EUSTACE-FISHER (c. 1212). La vie de Saint Eustache par Pierre Beauvais, éd. J. R. Fisher. Thèse de Ph.D., Columbia University, 1927.

eustace-Petersen (XIII ${ }^{\mathrm{e}}$ s.). La vie de Saint Eustache. Poème français du XIII ${ }^{e}$ siècle, éd. H. Petersen. Paris : Champion, 1928.

FROISSART (1373). Jehan Froissart, Chroniques. Dernière rédaction du premier livre, éd. G. T. Diller. Genève : Droz, 1972.

GORMONT (1125). Gormont et Isembart, éd. A. Bayot. Paris : Champion, 1931.

MARIE-DE-FRANCE (c. 1160). Les lais de Marie de France, éd. J. Rychner. Paris : Champion, 1973.

PERCEFOREST (c. 1330). Le roman de Perceforest. Première partie, éd. J. H. M. Taylor. Genève : Droz, 1979.

PRISE (c. 1370). Guillaume de Machaut. La prise d'Alexandrie, ou Chronique du roi Pierre Ier de Lusignan, éd. L. de Mas Latrie. Genève : Fick, 1877.

QUATRElivRe (c. 1170). Li quatre livre des reis, éd. E. R. Curtius. Gesellschaft für romanische Literatur, no. 9. Dresden, Halle : Max Niemeyer, 1911.

QUESTE (c. 1225). Queste del saint Graal, éd. C. Marchello-Nizia et A. Lavrentiev, Lyon, Equipe BFM, 2013. Publié en ligne par la Base de français médiéval, http://catalog.bfm-corpus.org/qgraal cm. Dernière révision le 2013-0719. Traduction par Isabelle Vedrenne-Fajolles (Université de Nice). Publication de l'équipe du projet BFM, ICAR ENS-LSH Lyon, 2005. http://bfm.ens-lsh.fr/queste-agreg.

RENART (c. 1180). Le Roman de Renart. Première branche, éd. M. Roques. Paris : Champion, 1948.

ROLAND (c. 1100). La chanson de Roland, éd. G. Moignet, $3^{\mathrm{e}}$ édition revue et corrigée. Paris : Bordas, 1972.

YVAIN (c. 1170). Les romans de Chrétien de Troyes, édités d'après la copie de Guiot (Bibl. nat. fr. 794), éd. M. Roques. Paris : Champion, 1960.

\section{Références bibliographiques linguistiques}

Batllori M., Iglésias, N. \& Martins, A. M. (2005). Sintaxi dels clítics pronominals en català medieval. Caplletra 38, Primavera, 137-177.

Benincà, P. (1995). Complement Clitics in Medieval Romance : The Tobler-Mussafia Law. In Battye, A. \& Roberts, I. (éds.), Clause Structure and Language Change, New York : Oxford University Press, 325-344.

Benincà, P. (2006). A Detailed Map of the Left Periphery of Medieval Romance. In Zanuttini, R., Campos, H., Herburger, E. \& Portner, P. (éds.), Crosslinguistic Research in Syntax and Semantics. Negation, Tense and Clausal Architecture, Washington : Georgetown University Press, 53-86.

Benincà, P., \& Poletto, C. (2004). Topic, Focus and V2 : Defining the CP sublayers. In Rizzi, L. (éd.), The structure of CP and IP : The Cartography of Syntactic Structures, vol. 2, Oxford : Oxford University Press, 52-75.

BFM - Base de Français Médiéval [En ligne]. Lyon : ENS de Lyon, Laboratoire ICAR, 2012, <http://bfm.enslyon.fr>. 
Cardinaletti, A. (2003). Stylistic Fronting in Italian. In Delsing, L.-O., Falk, C., Josefsson, G. \& Sigurðsson, H. Á. (eds.), Grammar in focus. Festschrift for Christer Platzack, Dept. of Scandinavian Languages, Lund : Wallin and Dalholm, 47-55.

Cardinaletti, A. \& Roberts, I. (1991/2002). Clause structure and X-second. In Cinque, G. (éd.), Functional Structure in DP and IP. The Cartography of Syntactic Structures, vol. 1, Oxford : Oxford University Press, 123-166. [manuscrit en circulation depuis 1991]

Cinque, G. (1999). Adverbs and Functional Heads : A Cross-linguistic Perspective. Oxford: Oxford University Press.

Dupuis, F. (1989). L'expression du sujet dans les subordonnées en ancien français. Thèse de Ph.D., Université de Montréal.

Egerland, V. (2011). Fronting, Background, Focus : A comparative study of Sardinian and Icelandic. Working Papers in Scandinavian Syntax, 87, 103-135.

Fanselow, G. \& Lenertová, D. (2011). Left peripheral focus : Mismatches between syntax and information structure. Natural Language and Linguistic Theory, 29.1, 169-209.

Fischer, S. \& Alexiadou, A. (2001). On Stylistic Fronting : Germanic vs. Romance. Working Papers in Scandinavian Syntax, 68, 117-145.

Fischer, S. (2010). Word order change as a source of grammaticalization. Amsterdam : John Benjamins.

Fischer, S. (2014). Revisiting Stylistic Fronting in Old Spanish. In Dufter, A. \& Octavio de Toledo, A. (éds), Left Sentence Peripheries in Spanish. Diachronic, Variationist and Comparative Perspectives, Philadelphia/ Amsterdam : John Benjamins, 53-75.

Fontana, J. M. (1993). Phrase Structure and the Syntax of Clitics in the History of Spanish. Thèse de Ph.D., University of Pennsylvania.

Franco, I. (2009). Verbs, Subjects and Stylistic Fronting. A comparative analysis of the interaction of CP properties with verb movement and subject positions in Icelandic and Old Italian. Thèse de Ph.D., Université de Sienne.

Franco, I. (2012). Verbal Stylistic Fronting in Old Florentine. http://ling.auf.net/lingbuzz/001510.

Frey, W. (2004). The grammar-pragmatics interface and the German prefield. Sprache und Pragmatik 52, 1-39.

Frey, W. (2006). Contrast and movement to the German prefield. In Molnár, V. \& Winkler, S. (éds.), The Architecture of Focus, Berlin : Mouton de Gruyter, 235-264.

Haegeman, L. (1997). Negative inversion and the structure of CP. Paper presented at the Linguistic Colloquium, University of Wuppertal.

Heycock, C. (2006). Embedded root phenomena. In Everaert, M. \& van Riemsdijk, H. (éds.), The Blackwell Companion to Syntax, vol. II, Oxford : Blackwell, 174-209.

Hirschbühler, P. (1995). Null Subjects in V1 Embedded Clauses in Philippe de Vigneulles' Cent Nouvelles Nouvelles. In Battye, A. \& Roberts, I. (éds.), Clause Structure and Language Change, Oxford : Oxford University Press, 257-291.

Hirschbühler, P. \& Junker, M-O. (1988). Remarques sur les sujets nuls en ancien et en moyen français. Revue Québécoise de Linguistique Théorique et Appliquée, 7.3, 63-84.

Holmberg, A. (2000). Scandinavian Stylistic Fronting : How Any Category Can Become an Expletive. Linguistic Inquiry, 31.3, 445-483.

Holmberg, A. (2006). Stylistic fronting. In Everaert, M., and van Riemsdijk, H. (éds), The Blackwell Companion to Syntax, Oxford : Blackwell, 532-565.

Holmberg, A. (2013, sous presse). Verb Second. In Kiss, T. and Alexiadou, A. (éds.), Syntax - an International Handbook of Contemporary Syntactic Research, Berlin : Walter de Gruyter Verlag.

Hrafnbjargarson, G. H. (2004). Stylistic Fronting. Studia Linguistica, 58.2, 88-134. 
Labelle, M. \& Hirschbühler, P. (2005). Changes in clausal organization and the position of clitics in Old French. In Batllori, M., Hernanz, M-L., Picallo, C. \& Roca, F. (eds.), Grammaticalization and Parametric Change, Oxford : Oxford University Press, 60-71.

Labelle, M. (2007). Clausal Architecture in Early Old French. Lingua, 117.1, 289-316.

Labelle, M. (2013). Participle fronting and clause structure in Old and Middle French. Communication présentée à LSRL 43, New York : CUNY Graduate Center.

Labelle, M \& Hirschbühler, P. (2005). Changes in clausal organization and the position of clitics in Old French. In Batllori, M. \& Roca, F. (éds), Grammaticalization and Parametric Change, Oxford : Oxford University Press, 60-71.

Maling, J. (1990). Inversion in embedded clauses in Modern Icelandic. In Maling, J. \& Zaenen, A. (éds.), Modern Icelandic Syntax, San Diego : Academic Press, 71-91. Réédition de Íslenskt mál, 1980. 2, 175-193.

Martineau, F., Hirschbühler, P., Kroch, A. \& Morin Y.-Ch. (2010). Corpus MCVF (parsed corpus), Modéliser le changement : les voies du français, Département de français, Université d'Ottawa. CD-ROM.

Martins, A. M. (2005). Clitic Placement, VP-ellipsis and scrambling in Romance. In Batllori, M., Hernanz, M.-Ll., Picallo, C. \& Roca, F. (éds), Grammaticalization and Parametric Change, Oxford \& New York : Oxford University Press, 175-193.

Martins, A. M. (2011). Scrambling and Information Focus in Old and Contemporary Portuguese. Catalan Journal of Linguistics 10, 133-158.

Mathieu, É. (2006a). Stylistic Fronting in Old French. Probus 18, 219-266.

Mathieu, É. (2006b). Quirky subjects in Old French. Studia Linguistica 60.3, 282-312.

Mathieu, É. (2007). À propos des propriétés germaniques de l'ancien français. Cahiers linguistiques d'Ottawa, 35, 107-136.

Mathieu, É. (2009). On the Germanic properties of Old French. In Crisma, P. \& Longobardi, G. (éds), Historical Syntax and Linguistic Theory, Oxford : Oxford University Press, 344-357.

Mathieu, É. (2013). The left periphery in Old French. In Arteaga, D. (éd.), Research in Old French : The State of the Art, Dordrecht : Springer, 327-350.

Moignet, G. (1979). Grammaire de l'ancien français. Paris : Klincksieck.

Molnár, V. (2010). Stylistic Fronting and Discourse. Tampa Papers in Linguistics, 1, 30-61.

Ott, D. (2009). Stylistic Fronting as Remnant Movement. Working Papers in Scandinavian Syntax, 83, 141-178.

Platzack, Ch. (1987). The Scandinavian Languages and the Null-Subject Parameter. Natural Language \& Linguistic Theory, 5.3, 377-401.

Poletto, C. (2005). Diachronic variation in Romance. Exemplier d'une conférence présentée à Eastern Generative Grammar summerschool, Wroclaw, Pologne. [http://egg.auf.net/05/docs/handouts/Cecilia/eggdiachronicpoletto. pdf].

Poletto, C. (2006). Parallel Phases : a study on the high and low left periphery of Old Italian. In Frascarelli, M. (éd.) Phases of Interpretation, Berlin : Mouton de Gruyter, 261-295

Prince, E. (1981). Topicalization, Focus-Movement, and Yiddish-Movement : A Pragmatic Differentiation. In Alford, D. (éd.), Proceedings of the Seventh Annual Meeting of the Berkeley Linguistics Society, Berkeley, CA : Berkeley Linguistic Society, 249-264. http://elanguage.net/journals/bls/article/view/2252

Rainsford, T., Guillot, C., Lavrentiev, A. \& Prévost, S. (2012). La position préverbale en ancien français : apport de corpus annotés, in Neveu, F. Muni Toke, V., Blumenthal, P., Klingler, T., Ligas, P., Prévost, S. \& TestonBonnard, S. (éds), Actes du 3e Congrès Mondial de Linguistique Française, 159-176.

Rickard, P. (1962). The Word-order Object-verb-subject in Medieval French. Transactions of the Philological Society, 61.1, 1-39.

Rivero, M-L. (1992). Clitic and NP climbing in Old Spanish. In Campos, H. \& Martínez-Gil, F. (éds.), Current Studies in Spanish Linguistics, Washington D.C. : Georgetown University Press, 241-282. 
Rizzi, L. (1997). The fine structure of the left periphery. In Haegeman, L. (éd.) Elements of Grammar : A Handbook of Generative Syntax, Dordrecht : Kluwer, 281-337.

Rizzi, L. (2005). On some properties of subjects and topics. In Brugé, L. Giusti, G., Munaro, N., Schweikert, W. \& Turano, G. (éds), Proceedings of the XXX Incontro di Grammatica Generativa, Venezia : Cafoscarina, 203-224.

Rizzi, L. (2006). On the Form of Chains : Criterial Positions and ECP Effects. In Cheng, L. \& Corver, N. (éds.), Wh Movement : Moving on, Cambridge, MA. : MIT Press, 97-134.

Rizzi, L., \& Shlonsky, U. (2006). Satisfying the Subject Criterion by a Non Subject : English Locative Inversion and Heavy NP Shift. In Frascarelli, M. (éd.), Phases of interpretation, Berlin : Mouton de Gruyter, 341-361.

Rizzi, L. \& Schlonsky, U. (2007). Strategies of subject extraction. In Sauerland, U. \& Gärtner, H-M. (éds.), Interfaces + Recursion = Language ? : Chomsky's Minimalism and the View from Syntax-Semantics, Berlin : Walter de Gruyter, 115-160.

Roberts, I. (1993). Verbs and Diachronic Syntax: A Comparative History of English and French. Dordrecht : Kluwer.

Roberts, I. (1999). The C-system in Brythonic Celtic Languages, V2 and the EPP. ms. University of Cambridge.

Rouveret, A. (2004). Les clitiques pronominaux et la périphérie gauche en ancien français. In Bulletin de la Société de Linguistique de Paris, t. XCIX, fasc. 1, 181-237.

Ruhnau, A. (2011). Interpretation of the Topological Field Model of the German Clause in Role and Reference Grammar. Masterprufung in Linguistik integrativ, Heinrich-Heine Universität Dusseldorf, Institut für allgemeine Sprachwissenschaft.

Salvesen, Ch. M. (2011). Stylistic Fronting and Remnant movement in Old French. In Romance Languages and Linguistic Theory. Selected Papers from 'Going Romance’ Nice 2009, Bern, J., Jacobs, H. \& Scheer, T. (éds.), Amsterdam : John Benjamins, 323-342.

Salvesen, Ch. M. (2013). Topics and the left periphery : a comparison of Old French and Modern Germanic. In Lohndal, T. (éd.) In Search of Universal Grammar. From Old Norse to Zoque, Amsterdam : John Benjamins, 131-171. (version préliminaire 2011. When V2 is disguised as V3 - a comparison of Modern Germanic and Old French, Université d'Oslo)

Sigurðsson, H. Á. (2010). On EPP effects. Studia Linguistica, 64.2, 159-189.

Sigurðsson, H. Á. (2013). On Stylistic Fronting again. Exemplier mis à jour d’un exposé fait à Tübingen, 2010.

Simonenko, A. \& Hirschbühler, P. (2012). Placement de clitiques dans les propositions V1 et évolution de la structure de la proposition en ancien français, in Dufresne, M. (éd.), Constructions en changement, Laval, Québec : Les Presses de l'université Laval, 11-53.

Taraldsen, K. T. (2001). Subject extraction, the distribution of expletives and stylistic inversion. In Hulk, A. \& Pollock, J.-Y. (éds.), Subject Inversion in Romance and the Theory of Universal Grammar, NewYork : Oxford University Press, 163-181.

Travis, L. (1984). Parameters and Effects of Word Order Variation. Thèse de Ph.D., Cambridge, MA: MIT.

Travis, L. (1991). Parameters of Phrase Structure and V2 Phenomena. In Freidin, R. (éd.), Principles and Parameters in Comparative Grammar, Cambridge, MA : MIT Press, 339-364.

Thráinsson, H. (2007). The Syntax of Icelandic. Cambridge : Cambridge University Press.

Vance, B. (1997). Syntactic Change in Medieval French : Verb second and null subjects. Dordrecht: Kluwer Academic Publishers.

Wood, J. (2011). Stylistic Fronting in spoken Icelandic relatives. Nordic Journal of Linguistics 34.1, 29-60.

Zaring, L., (2011a). On the nature of embedded V2 in Old French. Handout of a paper presented at the Diachronic Generative Syntax (DiGS) Conference 13. University of Pennsylvania, Philadelphia, PA, June 5, 2011.

Zaring, L. (2011b). On the nature of OV and VO order in Old French. Lingua 121, 1831-1852.

Zwart, C. J-W. (1993). Dutch Syntax - A Minimalist Approach. Thèse de Ph.D., Université de Groningen. 


\begin{abstract}
${ }^{1}$ Ces possibilités peuvent être expliquées dans une approche avec périphérie gauche étoffée, où la structure dans laquelle s'inscrit la construction V2 peut être coiffée d'un ou plusieurs niveaux. Dans ce type d'approche, les constructions où le verbe occupe une position plus reculée sont produites par la présence d'expressions additionnelles dans ces niveaux supérieurs, comme des expressions introduisant le cadre temporel, des expressions topicales, des éléments disloqués à gauche (voir, parmi d'autres, les travaux récents de Rouveret 2004, Labelle 2007, Salvesen 2011, Rainsford, Guillot, Lavrentiev \& Prévost 2012). Le cas où le verbe occupe la position initiale serait le résultat de l'antéposition du verbe dans un niveau supérieur à celui qu'il occupe dans une phrase V2 (Beninca 1995, Labelle \& Hirschbühler 2005, Simonenko \& Hirschbühler 2012).
\end{abstract}

${ }^{2}$ Dans les exemples, les éléments déplacés par l'antéposition stylistique (SF [stylistic fronting], pour conserver l'étiquette communément employée), ou le déplacement stylistique à gauche (DSG), sont soulignés et en bleu. La position source de l'expression déplacée est signalée par une trace co-indicée avec son antécédent. Lorsque cela est utile, les autres éléments de la phrase qui sont importants sont en caractères gras. Dans certains cas, pour la clarté, au lieu d'indiquer la position source de l'expression extraite par une trace, nous le faisons en laissant l'occurrence de départ, doublement barrée.

${ }^{3}$ Pour l'antéposition V2, Maling parle de 'topicalisation' en mentionnant qu'il peut s'agir d'un emploi impropre du terme, puisqu'il ne fait pas référence au rôle discursif de cet élément, mais qu'il s'agit plutôt d'un emploi bien établi (à l'époque) en syntaxe générative. On peut consulter Prince (1981) pour un historique des divers emplois de ce terme en grammaire générative dans la période 1970-1981.

${ }^{4}$ Sur la base d'observations faites par Holmberg (2006), cette hiérarchie modifie celle présentée initialement par Maling en plaçant les participe passés et particules verbales avant les adjectifs prédicatifs.

${ }^{5}$ La référence des exemples tirés du corpus MCVF (Martineau et coll. 2010) est celle donnée dans le corpus. Dans le cas de Roland, le premier chiffre correspond à la laisse.

${ }^{6}$ Les exemples (34)-(36) montrent que la négation ne bloque également pas l'antéposition dans d'autres langues romanes.

${ }^{7}$ Les exemples indiqués comme BFM proviennent de la Base de français médiéval, http://bfm.ens-lyon.fr/.

${ }^{8}$ Pour Sigurðsson (communication personnelle), un exemple comme (i) (= Holmberg 2006, ex. 26b), avec antéposition d'un complément sélectionné, n'est pas parfait, mais il considère que ce n'est pas une question de grammaticalité. Sigurðsson (2013, ex. 11c) considère ce type d'exemple comme similaire à des exemples qu'il marque '??’. Pour davantage sur le sujet, de “sem i Osló hafa búið”, voir Sigurðsson (2013, p. 3 sqq).

(1) peir sem í Ósló hafa búið à

'ceux qui à Oslo ont vécu'.

${ }^{9}$ Selon Mathieu (2006a : 254), le déplacement d'une tête sous Top ${ }^{0}$ n'est pas un mouvement de tête-à-tête, mais un déplacement long de tête (long head movement). 